\title{
Molecular identification, sequence analyses, tissue expression profile of a novel cattle gene-GBE1 in Yunnan cattle
}

\author{
G.Y. Liu ${ }^{1}$ \\ Key Laboratory of Animal Nutrition and Feed of Yunnan Province, \\ Yunnan Agricultural University \\ Kunming 650201, P.R. China
}

(Received 30 March 2008; revised version 17 June 2008; accepted 27 October 2008)

\begin{abstract}
The complete coding sequences of a cattle gene - the glucan (1,4-alpha-), branching enzyme 1 (glycogen branching enzyme), GBE1, was amplified using the reverse transcriptase polymerase chain reaction (RT-PCR) based on the conserved coding sequence information of the human, mouse and highly homologous cattle ESTs. Sequence analysis revealed that cattle GBE1 has high homology with the GBE1 from six species: dog (92\%), cat $(91 \%)$, horse $(91 \%)$, human $(89 \%)$, mouse $(88 \%)$ and rat $(88 \%)$. The phylogenetic tree analysis revealed that the cattle GBE1 has closer genetic relationships with the GBE1 of dog and cat. Tissue expression profile analysis revealed that the cattle GBE1 gene was highly expressed in liver, moderately expressed in muscle and fat, weakly expressed in kidney, small intestine, large intestine, heart and lung. Our experiment established the primary foundation for further research on the cattle GBE1 gene.
\end{abstract}

KEY WORDS: cattle, GBE1, tissue expression

\section{INTRODUCTION}

The glucan (1,4-alpha-), branching enzyme 1 (glycogen branching enzyme), GBE1, is one important monomeric enzyme functions in glycogen synthesis by catalyzing the formation of alpha 1,6-glucosidic linkages. It is most highly expressed in liver and muscle. Mutations in the gene coding GBE1 enzyme can result in glycogen storage disease IV (Andersen's disease) (Chan et al., 1999;

\footnotetext{
${ }^{1}$ Corresponding author: e-mail: liuyg4567@163.com
} 
Ziemssen et al., 2000; McCarthy et al., 2003; Bruno et al., 2007; Fyfe et al., 2007). Andersen's disease always leaded to the infants death, muscle hypotonia, retarded growth, splenomegaly, and progressive hepatomegaly (Gómez-Garre et al., 2007; Miyahara et al., 2007).

Based on above described, GBE1 gene is associated with glycogen synthesis, mutation disease of human and animals such as horse (Ward et al., 2003). As same as mammalian, this inherited, recessive disease also maybe occur in cattle. If this is a fact, it is essential to isolate this gene from cattle for this disease, potentially, is related to the cattle production (glycogen synthesis, mutation disease). If this disease was found in cattle, cattle would be another important kind of animal model of human to study this disease. Althrough a partial encoding sequence of the cattle GBE1 gene had been deposited into the NCBI database (GeneBank number: XM_867634), until today, the cattle GBE1 has not been reported yet.

In this study we isolated the coding sequence of cattle GBE1 gene, subsequently performed some necessary sequence analyses and tissue expression profile analysis for this gene. This will establish the primary foundation of understanding this cattle gene.

\section{MATERIAL AND METHODS}

Samples collection, RNA extraction and first-strand cDNA synthesis

The tissue samples of muscle, heart, liver, fat, kidney, lung, small intestine, large intestine, were derived from five adult Yunnan local cattles (all about 3 years old, female, healthy, housed in one cote and freely graze). Total RNA extraction and first-strand cDNA synthesis for these tissue samples were performed as the methods described by Liu et al. (2004).

\section{Isolation of the cattle GBE1 gene}

The RT-PCR was performed to isolate this cattle gene using the pooled cDNAs from different tissues mentioned above. The $25 \mu 1$ reaction system was: $2.0 \mu \mathrm{cDNA}(100 \mathrm{ng} / \mu \mathrm{l}), 2.5 \mu 12 \mathrm{mM}$ mixed dNTPs, $2.5 \mu 110 \times$ Taq DNA polymerase buffer, $2.5 \mu \mathrm{l} 25 \mathrm{mM} \mathrm{MgCl}, 2.0 \mu 110 \mu \mathrm{M}$ forward primer, $2.0 \mu 1$ $10 \mu \mathrm{M}$ reverse primer, 2.0 units of Taq DNA polymerase $(1 \mathrm{U} / 1 \mu \mathrm{l})$, and $9.5 \mu \mathrm{l}$ sterile water. The primers for cattle GBE1 gene isolation was designed based on the conserved CDS sequences information from human and mouse GBE1 gene (GeneBank numbers: L07956.1 and BC017541 ) and their highly homologous cattle EST sequences (GeneBank numbers: EE348093, DV917642, DV775425 and 
DY458736). The primer sequences and their annealing temperature for RT-PCR reaction were described in Table 1 . The PCR program initially started with a $94^{\circ} \mathrm{C}$ denaturation for $4 \mathrm{~min}$, followed by 35 cycles of $94^{\circ} \mathrm{C} / 1 \mathrm{~min}, \mathrm{Ta}^{\circ} \mathrm{C} / 1 \mathrm{~min}, 72^{\circ} \mathrm{C} / 1$ min, then $72^{\circ} \mathrm{C}$ extension for $10 \mathrm{~min}$, finally $4^{\circ} \mathrm{C}$ to terminate the reaction.

Table 1. Primers for cattle GBE1, G3PDH and annealing temperature

\begin{tabular}{llc}
\hline Gene & \multicolumn{1}{c}{ Primer sequence } & Ta $/{ }^{\circ} \mathrm{C}$ \\
\hline \multirow{2}{*}{ GBE1 } & $\begin{array}{l}\text { Forward: 5' - ATGGCGGCTCCGGCAGGT-3' } \\
\text { Reverse: 5' - TCAGTTGGGCAGGTCCAC-3' }\end{array}$ & 57 \\
\multirow{2}{*}{ G3PDH } & $\begin{array}{l}\text { Forward: 5' - ACCACAGTCCATGCCATCAC-3 } \\
\text { Reverse: 5' - AAGAAGGTGGTGAAGCAGG-3 }\end{array}$ & 55 \\
\hline
\end{tabular}

This PCR product for cattle GBE1 was then cloned into PMD18-T vector and sequenced bidirectionally with the commercial fluorometric method. At least five independent clones was sequenced.

\section{RT-PCR for tissue expression profile analysis}

RT-PCR tissue expression profile analyses was performed as previously described elsewhere (Liu et al., 2007). We selected the cattle housekeeping gene G3PDH (glyceraldehyde-3-phosphate dehydrogenase) (Accession number: AF077815) as the internal control. The control primers used are presented in Table 1. The PCR product is 148 bp in length. The primers of cattle GBE1 gene which were used to perform the RT-PCR for tissue expression profile analysis were the same as the primers for isolation RT-PCR above. The PCR reaction was optimized for a number of cycles to ensure product intensity within the linear phase of amplification. The $25 \mu 1$ reaction system was: $2 \mu 1$ pooled cDNA of each tissue $(100 \mathrm{ng} / \mu \mathrm{l}), 5 \mathrm{pM}$ each oligonucleotide primer, $2.5 \mu 12 \mathrm{mM}$ mixed dNTPs, $2.5 \mu 1$ 10×Taq DNA polymerase buffer, $2.5 \mu 125 \mathrm{mM} \mathrm{MgCl}_{2}, 1.0$ units of Taq DNA polymerase, and sterile water to the volume of $25 \mu$. The PCR program initially started with a $94^{\circ} \mathrm{C}$ denaturation for $4 \mathrm{~min}$, followed by 25 cycles of $94^{\circ} \mathrm{C} / 1 \mathrm{~min}, \mathrm{Ta}^{\circ} \mathrm{C} / 1 \mathrm{~min}, 72^{\circ} \mathrm{C} / 1 \mathrm{~min}$, then $72^{\circ} \mathrm{C}$ extension for $10 \mathrm{~min}$, finally $4^{\circ} \mathrm{C}$ to terminate the reaction.

\section{Sequence analysis}

The cDNA sequence prediction was conducted using GenScan software (http://gene.mit.edu/GENSCAN.html). The protein prediction and analysis were performed using the Conservedd Domain Architecture Retrieval Tool of BLAST at the National Center for Biotechnology Information (NCBI) server 
(http://www.ncbi.nlm.nih.gov/BLAST) and the ClustalW software (http://www.ebi. ac.uk/clustalw).

\section{RESULTS}

cDNA amplification of cattle GBE1 gene. Through RT-PCR with pooled tissue cDNAs from muscle, heart, liver, backfat, kidney, lung, small intestine, large intestine, for cattle GBE1, the resulting PCR product was 2118 bp in length (Figure 1).

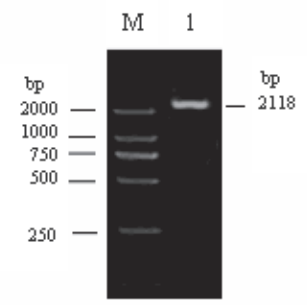

Figure 1. RT-PCR result for cattle GBE1. M, DL2000 DNA marker; 1, PCR product for cattle GBE1 gene

Sequence analysis. The cDNA nucleotide sequence analysis using the BLAST software at NCBI server (http://www.ncbi.nlm.nih.gov/BLAST) revealed that this gene was not homologous to any of the known cattle gene and it was then deposited into the GenBank database (Accession number: EU442575). The sequence prediction was carried out using the GenScan software and results showed that the 2118bp cDNA sequence represented a single gene which encoded 705 amino acids. The complete CDS of this gene and the encoded amino acids was presented in Figure 2.

Further BLAST analysis of this protein revealed that cattle GBE1 has high homology with the glucan (1,4-alpha-), branching enzyme 1 (glycogen branching enzyme), GBE1, from six species - dog (92\%), cat (91\%), horse (91\%), human $(89 \%)$, mouse $(88 \%)$ and rat $(88 \%)$ (Figure 3$)$.

Based on the result of the alignment of GBE1 the phylogenetic tree was constructed using the ClustalW software (http://www.ebi.ac.uk/clustalw), as shown in Figure 4. The phylogenetic tree analysis revealed that the cattle GBE1 has closer genetic relationship with the GBE1 of dog and cat.

Tissue expression profile. Tissue expression profile analysis was carried out and results revealed that, compared to G3PDH expression, the cattle GBE1 gene was highly expressed in liver, moderately expressed in muscle and fat, weakly expressed in kidney, small intestine, large intestine, heart and lung (Figure 5). 
ATGGCGGCTCCGGCAGGTCTCCCGGCTGAGGCGGCTGGGGCCGAATGCTCTGAGGAGGCGCTAGCC

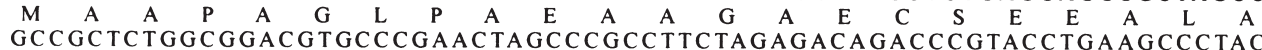
$\begin{array}{llllllllllllllllllllll}A & A & L & A & D & V & P & E & L & A & R & L & L & E & T & D & P & Y & L & K & P & Y\end{array}$ GCTCCGGACT TCCAGCGCAGGTATAAGCGGTTTAACCAGACT T TGACCGACATTGGAGAGAATGAA $\begin{array}{llllllllllllllllllllll}A & P & D & F & Q & R & R & Y & K & R & F & N & Q & T & L & T & D & I & G & E & N & E\end{array}$ GGTGGTAT TGATAGGTTTTCCAGAGGTTATGAATCATTTGGCGTCCACAGATGC GCTGATGGTGGTTTA $\begin{array}{lllllllllllllllllllllll}G & G & I & D & R & F & S & R & G & Y & E & S & F & G & V & H & R & C & A & D & G & G & L\end{array}$ TAC TGCAAAGAATGGGCCCCAGGAGCTGAAGGAGT T T T TCT TACTGGAGACT TCAATGATTGGAAC $\begin{array}{llllllllllllllllllllll}Y & C & K & E & W & A & P & G & A & E & G & V & F & L & T & G & D & F & N & D & W & N\end{array}$ CCATT T T ATACCCATATAAAAAACTGGATTATGGAAAATGGGAGCTGTATATCCCACCAAAGCAAAAT $\begin{array}{lllllllllllllllllllllll}P & F & S & Y & P & Y & K & K & L & D & Y & G & K & W & E & L & Y & I & P & P & K & Q & N\end{array}$ AGATCTGTCTTAGTACCTCATGGATCCAAACTAAAGCTTGTTATCAGGAGTAAAAGTGGAGAAATCT TG

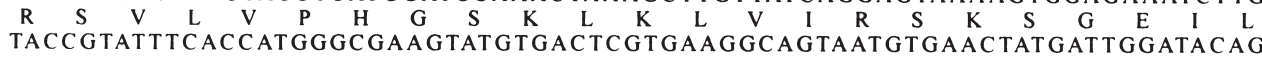
$\begin{array}{llllllllllllllllllllllll} & R & I & S & P & W & A & K & Y & V & T & R & E & G & S & N & V & N & Y & D & W & I & Q\end{array}$ TGGGATCCAGAATACTCGTATAAATTTAAACATTCCAAACCAAAGAAGCCAAAAGGTCTACGAATTTAT $\begin{array}{lllllllllllllllllllllll}W & D & P & E & Y & S & Y & K & F & K & H & S & K & P & K & K & P & K & G & L & R & I & Y\end{array}$ GAATC TCACGTGGGAAT T TCT TCC TATGAAGGAAAAATAGCT TCT TATAAACATTTTACATGCAATGTA

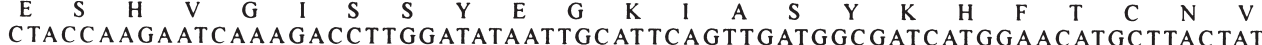

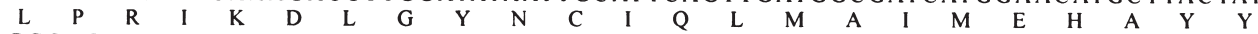
GCCAGT TT TGGTTACCAAATCACAAGCTTCT T TGCAGCT TCAAGCCGT TATGGAACTCCTGAAGAG

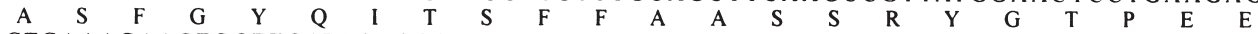
CTGAAAGA AC TGGTTGATACAGCCCATTCCATGGGTATTACAGTGCTCTTAGATGTGGTACACAGCCAT $\begin{array}{lllllllllllllllllllllll}\mathrm{L} & \mathrm{K} & \mathrm{E} & \mathrm{L} & \mathrm{V} & \mathrm{D} & \mathrm{T} & \mathrm{A} & \mathrm{H} & \mathrm{S} & \mathrm{M} & \mathrm{G} & \mathrm{I} & \mathrm{T} & \mathrm{V} & \mathrm{L} & \mathrm{L} & \mathrm{D} & \mathrm{V} & \mathrm{V} & \mathrm{H} & \mathrm{S} & \mathrm{H}\end{array}$ GCCTCAAAAAAT TCAGAAGATGGACTAAATATGTT TGACGGGACTGAATCCTGTTACT T TCATTATGGA

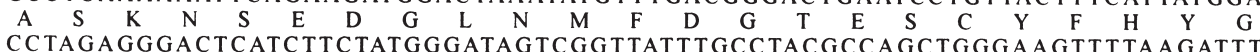

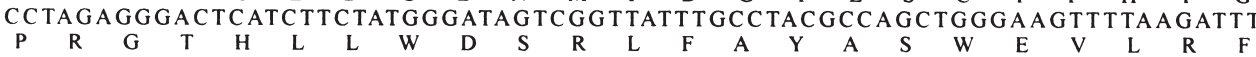
CTTCTGTCAAACATAAGATGGTGGTTGGAAGAGTATGGCTTTGATGGAT TTCGTTTTGACGGTGTTACA $\begin{array}{lllllllllllllllllllllll}\mathrm{L} & \mathrm{L} & \mathrm{S} & \mathrm{N} & \mathrm{I} & \mathrm{R} & \mathrm{W} & \mathrm{W} & \mathrm{L} & \mathrm{E} & \mathrm{E} & \mathrm{Y} & \mathrm{G} & \mathrm{F} & \mathrm{D} & \mathrm{G} & \mathrm{F} & \mathrm{R} & \mathrm{F} & \mathrm{D} & \mathrm{G} & \mathrm{V} & \mathrm{T}\end{array}$

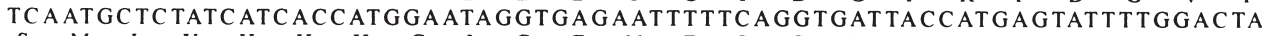
$\begin{array}{lllllllllllllllllllllll}S & M & L & Y & H & H & H & G & I & G & E & N & F & S & G & D & Y & H & E & Y & F & G & L\end{array}$ CAAGTAGATGAAGATGCTTTGACT TACATCATGTTGGCAAATCATTTGGTTCACACACTGTATCCAGAT $\begin{array}{lllllllllllllllllllllll}Q & \mathrm{~V} & \mathrm{D} & \mathrm{E} & \mathrm{D} & \mathrm{A} & \mathrm{L} & \mathrm{T} & \mathrm{Y} & \mathrm{I} & \mathrm{M} & \mathrm{L} & \mathrm{A} & \mathrm{N} & \mathrm{H} & \mathrm{L} & \mathrm{V} & \mathrm{H} & \mathrm{T} & \mathrm{L} & \mathrm{Y} & \mathrm{P} & \mathrm{D}\end{array}$ TCTATAACAATAGCTGAGGATGTATCCGGAATGCCAGCTCTATGT TC TCCAATT TCCCAGGGAGGGGGT

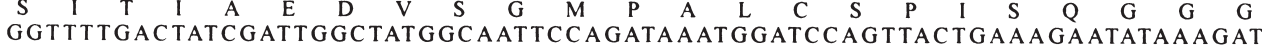

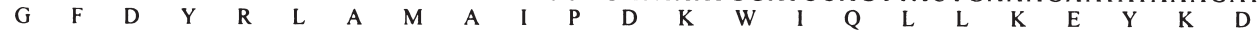
GAAGAT TGGAACATGGGGAATATAGTGTATACACTCACAAACCGACGCTACCTTGAAAAGTGCATT $\begin{array}{llllllllllllllllllllll}E & D & W & N & M & G & N & I & V & Y & T & L & T & N & R & R & Y & L & E & K & C & I\end{array}$ GCCTACGCAGAGAGCCATGATCAGGCACT TGTTGGGGATAAGACGT TGGCATTTTGGTTGATGGAT $\begin{array}{llllllllllllllllllllll}A & \mathrm{Y} & \mathrm{A} & \mathrm{E} & \mathrm{S} & \mathrm{H} & \mathrm{D} & \mathrm{Q} & \mathrm{A} & \mathrm{L} & \mathrm{V} & \mathrm{G} & \mathrm{D} & \mathrm{K} & \mathrm{T} & \mathrm{L} & \mathrm{A} & \mathrm{F} & \mathrm{W} & \mathrm{L} & \mathrm{M} & \mathrm{D}\end{array}$ GCTGAAATGTATACCAACATGAGCGTTCTGACCCCATT TACTCCCGTTATTGATCGTGGAATACAGCTT

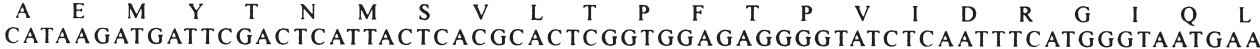

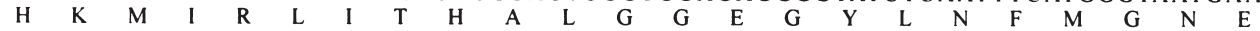
TTTGGGCATCCTGAATGGTTAGACTTCCCCAGAAAAGGAAATAATGA GA GCTACCATTATGCACGA

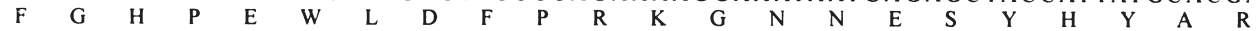
AAGCAGTTTCATTTAACTGATGATGACCTTCTTCGCTATAAGT TCCTAAATAACT T TGACAGGGATATG $\begin{array}{lllllllllllllllllllllll}K & Q & F & H & L & T & D & D & D & L & L & R & Y & K & F & L & N & N & F & D & R & D & M\end{array}$ AATAAATTGGAAGAAAGATGTGGTTGGCTTTCAGCTCCACAGGCCCATGTGAGTGAAAAGCATGAA

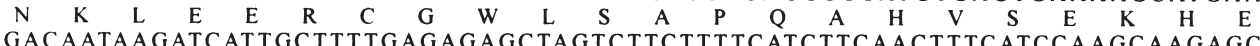
$\mathrm{D} \quad \mathrm{N} \quad \mathrm{K}$ I I I A $\mathrm{F}$ E $\mathrm{R}$ A $\mathrm{S}$ L L F I F N F H P S K S TATACTGATTACCGAGTTGGAACAACATTGCCAGGAAAGTACAAAAT TGTGCTCGATTCAGATGCA $\begin{array}{lllllllllllllllllllllll}Y & T & D & Y & R & V & G & T & T & L & P & G & K & Y & K & I & V & L & D & S & D & A\end{array}$ GCAGAATACGGAGGACACAAGAGACTGGACCACAATACCGAAT TC T TCTCTGAACCT T T TGAACAT

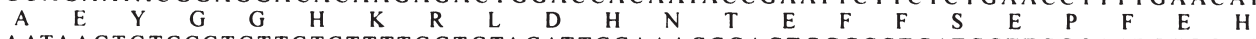
AATAACTGTCCCTGT TC TCT TT TGGTGTACAT TCCAAACCGAGTGGCCCTCATCCTTCGCAATGTGGAC $\begin{array}{lllllllllllllllllllllll}N & N & C & P & C & S & L & L & V & Y & I & P & N & R & V & A & L & I & L & R & N & V & D\end{array}$ CTGCCCAACTGA

$\mathrm{L} \mathrm{PN}^{*}$

Figure 2. The complete coding sequence of cattle GBE1 gene and its encoding amino acids * indicates the stop codon 
Dog

Cat

Cattle

Horse

Human

Mouse

Rat

Dog

Cat

Cattle

Horse

Human

Mouse

Rat

Dog

Cat

Cattle

Horse

Human

Mouse

Rat

Dog

Cat

Cattle

Horse

Human

Mouse

Rat

Dog

Cat

Cattle

Horse

Human

Mouse

Rat

Dog

Cat

Horse

Human

Mouse

Rat

Dog

Cat

Catele

Horse

Human

Mouse

Rat

Dog

Cat

Cattle

Horse

Human

Mouse

Rat
Cattle

\begin{abstract}
- - - MAAPAARAD CSEEALAAALADVPELARL LE ID PYLKP FAPDFQRRYKRFNETLN MAAPVARGE CSEAALAAALADVPELARL LELDPYLKPFALDFORRYKK FIETLN MAA PAGL PAEAAGAE C SEEALAAALADVPE LARL LETD PYLKPYA PDFQRRYKRFNQTLT - - - - MAA PAARAD GSDAALAAALADVPDL GRL LEVD PYLKPYAPD FQRRYMRFSOTLD - - MAAPMT PAARPED YEAALNAALADVPE LARL LEID PYLKPYAVDFORRYKOFSOILK - - MAA PAA PAAGETGPDARLEAAL ADVPE LARL LE ID PYLKP FAAD FORRYKKFIOVLH - -MAAPAAPAAEEKGSEAQLKAALADVPELGRL LE ID PYLKPYAADFQRRYKKFNQVLH : * *

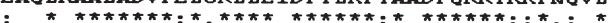

NIGENEGGIDKFSRGYE $\$$ FGVHRCADGGLYCKEWAPGAEGVFLTGD FNDWNP FSYPYKKL NI GENEGGIDKFSRGYE $\$$ FGVHRCADGGLYCKEWAP GAE GVFLTGD FNDWNP F $Y Y P Y K K L$ DIGENEGGIDRFSRGYES FGVHRCADGGL YCKTWAP GAE GVFLTGD FNDWNP FSYPYKKI NI GKINEGGIDKF \$RGYE $\$$ FGVHRCADGGLYCKEWA P GAEGVFLTGD FNDWN P FSYPYKKL NI GENE GGIDKF $\$$ RGYE $\$$ FGVHRCAD GGL YCKEWA P GAE GV FLTGD FNGWNP F $\$$ Y PYKKL DI GENEGGIDKF SRGYE $\$$ FGIHRCSD GGIYCKRWAP GAE GV FL TGE FS GUNP FSHPYKKL DI GENEGG IDKFSRGYE S FGIHRC SD GGIYCKEWAP GAE GVFLTGE FSGUNP FSHPYKKL

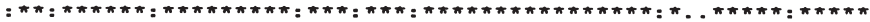

DYGKWE L YI P PKONKS L L VPHGSKLKVV IRSKSGE I L YRI S PWAKYVTREGDNVNYDWIH DY GKWE L Y I P PKQNKSQL VPHGSKLKVVIRSKS GE I L YRI S PWAKYVTRE GENVNYDWTH DYGKWE L Y I P PKONRSVL VPHG SKLKL VIRSKSGE I L YRI I PWAKYVTREG SNVNYDWIO DYGKWD L Y I P PKPNKS L L VPHGSKLKVV IRSKSGE I L YRI S PWAKYVVRES GNVNYDW IH DY GKWE L Y I P PKONKSVL VPHGSKLKVVITSKSGE I L YRI S PWAKYVVREGDNVNYDWIH EYGKWE L Y I P PKONKS P L I PHGSKLKVV ITSKSGE I L YR I S PWAKYVVRENNNVNYDW IH EY GKWE L Y I P PKONKS P P I PHGSKLKVVITSKSGE I L YRI S PWAKYVVRENNNVNYDWIH

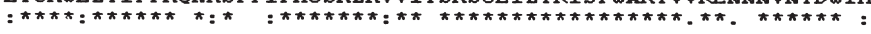

WD PEHPYKFKHSKPKKPRGLRIYESHVGIS SYEGKIASYKHFTCNVL PRIKDLGYNCIQL WD PEHPYKFKHSRPKKPRGVRIYESHVGISSYE GKIASYKHFTYNVL PRIKDL GYNCIOM WD PEYSYKFKHSKPKKPKGLRIYESHVGISSYEGKIASYKHFTCNVL PRIKDL GYNCIOL WD PEO PYKFKHSR PKKPRSLRIYE SHVGISSHEGKIASYKHFTCNVL PRIKGLGYNCIQM WD PEHSYE FKHSR PKKPRS LRIYE SHVGIS SHE GKVASYKHFTCNVL PRIKGLGYNCIQL WAPED PYKFKHSR PKRPRS LRIYESHVGISSHE GKIASYKHFTSNVL PRIKD L GYNCIOL WD PEN PYKFRHSR PKKPRS LRIYE SHVGIS SHE GKIASYKHFTSNVL PRIKD L GYNCIOL

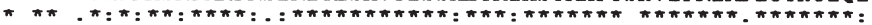
MAIMEHAYYAS FGYOITS FFAAS SRYGTPEE LKE L IDTAHSMGITVL LDVVHSHASKNSE MAIMEHA YYAS FGYOITS FFAAS SRYGT PEE LKE LVDTAHSMGITVL LDVVHSHASKNSE MA IMEHA YYAS FGYOITS FFAAS SRYGTPEE LKE L VDTAHSMGITVL LDVVHSHASKASE MAIMEHAYYASFGYOITSFFAAS SRYGTPEELKELVDTAHSMGITVLLDVYHSHASKNSE MAIMEHAYYAS FGYOITS FFAAS SRYGTPEE LOE LVDTAHSMGI IVL LDVVHSHASKNSA

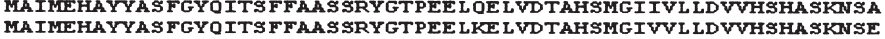
MA IMEHAYYA FGYOITS FFAAS SRYGTPEE LKE LVDTAHSMGIVVL LDVVHSHA.SKNSE

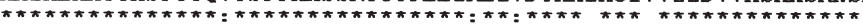

DGLNMFD GTD SCYFHSGPRGNHD LWD SRL FAYS SWEVLRFL L SNIRWWLEEYYFDGFRFD DGLNMFD GTD SCY FHS GPRGNHD LWD SRL FIY \$ SUEVLRFL L SNIRWWLEEYG FDGFR FD DGLNMFD GTD SCY FHS GPRGNHD LWD \$RL FIY S SWEVLRFL L SNIRUWLEEYGFDGFRFD DGLNMFD GTE SCY FHYGPRGTHL LUD SRL FAYASUEVLRFL L SNIRWWLEEYGFDGFRFD DGLNMFD GTD SCY FHS G PRGTHDLWDSRL FAY S SWEVLRFL L SNIRWWLEE YRFDG FRFD DGLNMFD GTD CY FHSGPRGTHD LWD \$RL FIYSSWEVLRFLL SNIRWWLEEYCFDGFRFD DGLNMFD GTD S CYFHS GPRGTHD L WD SRL FIYSSWEVLRFL L SNIRWWLEEYC FDGFRFD

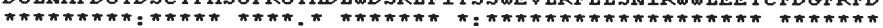

GVTSML YHHHGMGE GFS GD YHEY FGL QVDEDAL VYLMLANHL VHTL Y PDS ITVAEDVSGM GVTSML YHHHGMGOA FS GD YHEY FGL OVDEDA L I YLMLANHL VHTL Y PNS ITIAEDVSGM GVTSML YHHHGI GEN FS GD YHEY FGL QVDEDAL TY IMLANHL VHTL Y PDS ITIAEDVSGM GVTSML YHHHG I GAS F\$GD YHEY FGL OVDEDALTYLMLANHL VHTL Y PDSITIAEDVSGM GVTSML YHHHGVGOGFSGDY SEYFGLOVDEDALTYLML ANHLVHTLC PD ITIAEDVSGM GVTSML YHFHGMGOGFSGDYNEY FGLOVDEDAL IYLMLANHLAHTL Y PD ITIAEDVSGM GVTSML YHHHGMGOGFSGDYSEYFGL OVDEDAL VYLMLANHL THTMY PDS ITIAEDVSGM

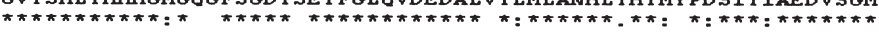

PALCSPISOGGVGFDYRLAMAI PDKWIOLLKE FKDEDWNMGNIVYTL TNRRYLEKCIAYA PALC P I SOGGVGFDYRLAMAI PDKWIOLLKE FKDEDUNMGGNIVYTLTNRRYLEKCIAYA PALCSPI OOGGGGFDYRLAMA I PDKWIOL LKTYRDEDINNMGNIVYTLTNRRYLEKCIAYA PALCSPI\$OGGGGFD YRLAMA I PDKWIOL VKE FKDEDWNMGNIVYTLTNRRHLEKCIAYA PALCSPISOGGGGFD YRLAMAI PDKWIQL LKE FKDEDWNMGD IVYTL TNRRYLEKC IAYA PALCSPTSOGGGGPDYRLAMA I PDKWIOL LKE FKDEDWNMGNIVYTL TNRRYLEKCVAYA PAL C SPTSOGGGGFDYRLAMAI PDKWIQL LKT FKDEDWNMGNIVYTLTNRRHLEKCVAYA

Figure 3. The alignment of the proteins encoded by GBE1 genes of cattle and other six species (continued on the page 497) 


Dog
Cat
Cattle
Horse
Human
Mouse
Rat
Dog
Cat
Cattle
Horse
Human
Mouse
Rat

Dog
Cat
Cattle
Horse
Human
Mouse
Rat

Dog
Cat
Cattle
Horse
Human
Mouse
Rat

Figure 3. continued
ESHDOALVGDKTLAFWLMDAEMYTNMSVLTP FTPVIDRGIOLHRMIRL ITHAL GGEGYLN ESHDOALVGDKTLAFULMDAEMYTNMSVLTP FTPVIDRGIOLHIMIRL ITHALGGEGYLN E \$HDOALVGDKTLAFWLMDAEMYTNMSVLTP FTPVIDRGIOLHKMIRL ITHAL GGEGYLN ESHDOALVGDKS LAFWLMDAEMYTNMSVLTP FTPVIDRGI QLHRMIRL ITHALGGEGYLN ESHDOALVGDKS LA FULMDAFMYTNMSYLTP FTPVIDRGIOLHRMIRI TTHGLGGEGYLN ESHDQALVGDKTLAFWLMDAEMYTNMSVLAP FTPVIDRGIQLHKMIRLITHGLGGEGYLN ESHDQALVGDKTLAFWLMDAEMYTMMSVLAP FTPVIDRGIQLHKMIRL ITHGLGGEGYLN
ESHDQALVGDKTLAFWLMD AEMYTNMSVLAP FTPVIDRGIQLHIKMIRL ITHGLGGEGYLN

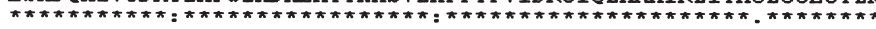

FMGNE FGHPEWLD FPRKGNNE SYHYARRO FHLTDDD L LRYKFLNN FDRDMINLEERCGWL FMGNE FGHPEWLD FPRKGNNE SYHYARRO FHL TDDDL LRYKFLNN FDRDMINLEERCGWL FMGNE FGHPEWLD FPRKGNNE SYHYARKO FHL TDDD L LRYKFLNN FDRDMNKLEERC GWL FMGNE FGHPEWLD FPRKGNNE SYHYARRO FHL TDDD L LRYKFLNN FDRDMNKLEERCGWL FMGNE FGHPEWLD FPRKGNNE SYHYARROFI FMGNE FGHPEWLD FPRKGNNE SYHYARRO FNL TDDDL LRYKFLNN FDRDMNRLEERCGWL FMGNE FGHPEWLD FPRKGNNE SYHYARRO FNL TDDD L LRYKFLNN FDRDMNRLEERCGWL

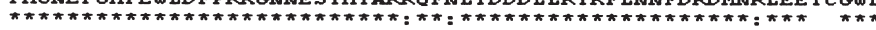

SAPOAYVSEKHEGNKI IAFERAGL L FI FN FHPSKSYTDYRVGTTL PGKY P FCCCRI IVLD SA POAFVSEKHEGNKI IAFERAGLVFI FNFHPSKSYTD YRVGTTL P GKFR---- - IVLD SA POAHV SEKHIEDNKI IA FERA L L FI FN FHP SKSYTD YRVGTTL P GKYK- - - - - IVLD SAPOAFV SEKTEGNKVIAFTRAAL I FI FNFHPSKSYTVYRVGTTI PGKFT-- - - IVLD AAPOAYVSEKTEGNKIIAFTRAGL FIFNFHPSTYTPYRVGTALPGKFT---- IVLD SAPQAYVSEKHEANKTITFERAGLL FIFN FHPSKSYTD YRVGTATPGKFK----- IVLD SAPQAYV SEKHEANKTITFERAGL L FI FN FHP SKSYTD YRVGTATPGKFK----- IVLD

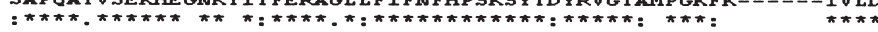

TDAAEYGGHORLDHNTD FFSED FKHINERP FS L L VYI P SRVGL IL ONVDMPN TDAAEYGGHORLDHSTE FFSO P FKHINER PC S L LVYI PNRVGL ILONVDMPN SDAAEYGGHKRLDHNTE FFSE P FEFDNNC PCS L L VYI PNRVAL ILRNVDL PN SDAAEYGGHORLDFNTD FF SE PYEHNERP S S L L VYI P SRVAL IL ONVD P PN SDAAEYGGHORLDHSTD FFSEAFEHNGRPYSLLVYI PSRVAL ILONVDL PN SDAAFYGHORLDHSTDFFEAFEHNGPYSLLVYIPSRVALILONVDLPN SDAARYGGHORLDHNTWYFALAFEFNGRPYSLLVYIPSRVALILONVDLON SDAAFYGGHORLDHSTDYFAEAFEHNGRPYSLL VYI PSRVAL IL QNVDL PN

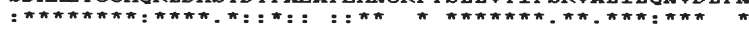

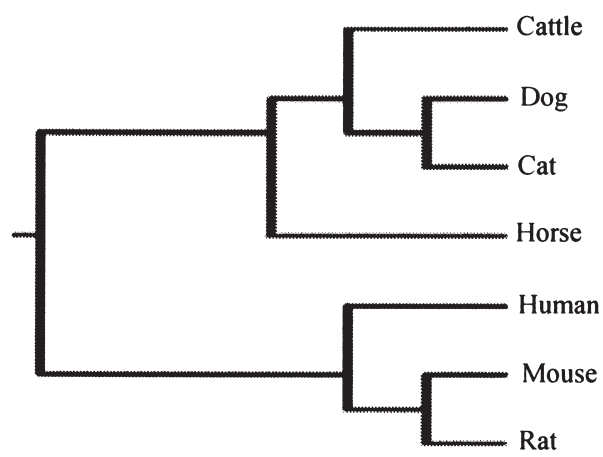

Figure 4. The phylogenetic tree for seven kinds of GBE1

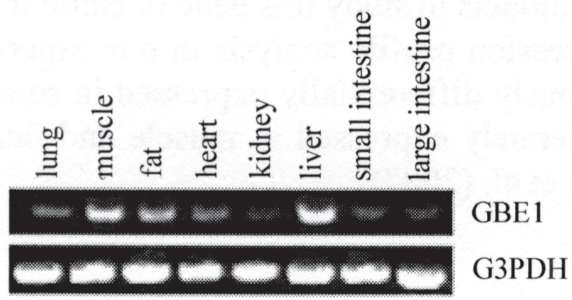

Figure 5. Tissue expression of cattle GBE1 gene. The G3PDH expression is the internal control 


\section{DISCUSSION}

Tay et al. (2004) had reported two single deletions (one in exon 10 and another in exon 12 ) in the CDS of GBE1 gene were associated with the human Andersen's disease. Wagner et al. (2006) also had reported a nonsense mutation in codon 34 of the GBE1 gene was associated with the horse Andersen's disease. Therefore, isolation of the coding regions of the GBE1 gene is utmost important to study this kind of disease.

Comparative genomics is the analysis and comparison of genomes from different species. Researchers have learned a great deal about the function of human gene by examining their counterparts in simpler model organisms such as the mouse and some results has revealed that virtually all (99\%) of the protein-coding gene in humans align with homologues in mouse, and over $80 \%$ are clear $1: 1$ orthologs (Hardison et al., 2003). This extensive conservation in protein-coding regions implied that this conservation of protein-coding sequences may be expected in different mammals including cattle. From the isolation of cattle GBE1 gene, we can find that cattle GBE1 is highly homologous with GBE1 of human, mouse and other mammals. This further validated that comparative genomics method is one useful tool to isolate the unknown gene especially the conserved coding region of gene for cattle.

From the alignment analyses for GBE1 proteins we found that no differences were detected at above mentioned loci associated with the glycogen storage disease IV in human, cattle, mouse and other mammals. This implied that the polymorphism of GBE1 gene at above mentioned loci may not depend on species but on individuals. These also implied that in the future research of this new gene, we should pay more attentions to that if above reported mutations would also happen in different cattle individuals and the potential associations of them with the cattle glycogen storage disease IV. For only five cattles were studied in this experiment, we think, more cattles are needed to study for the further detection.

The phylogenetic tree analysis revealed that the cattle GBE1 has closer genetic relationship with the GBE1 of dog and cat. This implied that dog and cat should be better animal models to study this gene of cattle than others.

From the tissue expression profile analysis in our experiment it can be seen that this gene was obviously differentially expressed in some tissues and highly expressed in liver, moderately expressed in muscle and fat. These agree to the results obtained by Fyfe et al. (2007). 


\section{CONCLUSIONS}

In conclusion, we first isolated the cattle GBE1 gene and performed some necessary analyses. This established the primary foundation for further research on this gene in cattle.

\section{REFERENCES}

Bruno C., Cassandrini D., Assereto S., Akman H. O., Minetti C., Di Mauro S., 2007. Neuromuscular forms of glycogen branching enzyme deficiency. Acta Myol. 26, 75-78

Chan Y.J., Lin S.P., Chen B.F., 1999. Glycogen storage disease type IV: a case report. Zhonghua Yi Xue Za Zhi (Taipei) 62, 743-747

Fyfe J.C., Kurzhals R.L., Hawkins M.G., Wang P., Yuhki N., Giger U., Van Winkle T.J., Haskins M.E., Patterson D.F., Henthorn P.S., 2007. A complex rearrangement in GBE1 causes both perinatal hypoglycemic collapse and late-juvenile-onset neuromuscular degeneration in glycogen storage disease type IV of Norwegian forest cats. Mol. Genet. Metab. 90, 383-392

Gómez-Garre P., Gutiérrez-Delicado E., Gómez-Abad C., Morales-Corraliza J., Villanueva V.E., Rodríguez de Córdoba S., Larrauri J., Gutiérrez M., Berciano J., Serratosa J.M., 2007. Hepatic disease as the first manifestation of progressive myoclonus epilepsy of Lafora. Neurology 68, $1369-1373$

Hardison R.C., 2003. Comparative genomics. PLoS Biol. 1, E58 Vol. 1, No. 2, e58 doi:10.1371/ journal.pbio.0000058

Liu G.Y., Xiong Y.Z., 2007. Isolation, sequence analysis and expression profile of a novel cattle gene, NIP7, differentially expressed in the Longissimus dorsi muscle tissues from Meishan, Meishan x Large White cross and Large White cattles. Mol. Biol. Rep. 34, 213-219

Liu Y.G., Xiong Y.Z., Deng C.Y., Zuo B., Zhang J.H., 2004. Comparison of gene expression patterns in Longissimus dorsi of cattles between the high-parent heterosis cross combination Landrace $\times$ Large White and the mid-parent heterosis cross combination Large White $\times$ Meishan. Asian-Austr. J. Anim. Sci. 17, 1192-1196

McCarthy J.J., Meyer J., Moliterno D.J., Newby L.K., Rogers W.J., Topol E.J., 2003. GenQuest multicenter study. Evidence for substantial effect modification by gender in a large-scale genetic association study of the metabolic syndrome among coronary heart disease patients. Hum. Genet. 114, 87-98

Miyahara A., Sugie H., 2007. Anderson disease/chylomicron retention disease. Nippon Rinsho 65, 597-599

Tay S.K., Akman H.O., Chung W.K., Pike M.G., Muntoni F., Hays A.P., Shanske S., Valberg S.J., Mickelson J.R., Tanji K., DiMauro S., 2004. Fatal infantile neuromuscular presentation of glycogen storage disease type IV. Neuromuscular Disord. 14, 253-260

Wagner M.L., Valberg S.J., Ames E.G., Bauer M.M., Wiseman J.A., Penedo M.C., Kinde H., Abbitt B., Mickelson J. R., 2006. Allele frequency and likely impact of the glycogen branching enzyme deficiency gene in Quarter Horse and Paint Horse populations. J. Vet. Intern. Med. 20, 1207-1211

Ward T.L., Valberg S.J., Lear T.L., Guérin G., Milenkovic D., Swinburne J.E., Binns M.M., Raudsepp T., Skow L., Chowdhary B.P., Mickelson J.R., 2003. Genetic mapping of GBE1 and its association with glycogen storage disease IV in American Quarter horses. Cytogenet. Genome Res. 102, 201-206

Ziemssen F., Sindern E., Schröder J.M., Shin Y.S., Zange J., Kilimann M.W., Malin J.P., Vorgerd M., 2000. Novel missense mutations in the glycogen-branching enzyme gene in adult polyglucosan body disease. Ann. Neurol. 47, 536-540 